\title{
NATIVE DEFECTS IN GALLIUM ARSENIDE GROWN BY SYNTHESIS, SOLUTE DIFFUSION METHOD
}

\author{
K. Frone, A. Mąrosa and T. Wosiński \\ Institute of Physics, Polish Academy of Sciences \\ Al. Lotników 32/46, 02-668 Warszawa, Poland
}

\begin{abstract}
High-purity $n$-type GaAs crystal was grown by the Synthesis, Solute Diffusion (SSD) method. Deep Level Transient Spectroscopy (DLTS) characterization of the crystal revealed three deep traps related to native defects. Microscopic origin of the traps is discussed and prospective use of SSD-grown GaAs as a bulk material with the high luminescence efficiency is emphasized.
\end{abstract}

PACS numbers: $61.70 .-r, 71.55 . \mathrm{Eq}$

\section{Introduction}

In this paper we report the results of native defect investigation in the first GaAs crystal grown in our laboratory by the Synthesis, Solute Diffusion (SSD) method. The method, which consists in synthesis from gallium solution, was adapted to GaAs growth by Kobayashi and Osaka [1]. It combines the advantage of low growth temperature, comparable to that of liquid-phase epitaxy (LPE), with the capability for production of large ingots. Despite its potential facility for the synthesis of high-purity bulk GaAs single crystals the method has not been applied on a large scale so far, the main reason being its very low growth rate, of about few mm/day, which is determined by arsenic diffusion in the gallium melt.

\section{Crystal growth and characterization}

GaAs crystal was synthesized in a quartz crucible placed in an evacuated quartz ampoule contained an arsenic source. The ampoule was set in a two-zone vertical furnace; the upper zone determining a temperature of gallium solution whereas the lower one controlling an arsenic pressure. The synthesis was performed 
without seed, at a temperature of $860^{\circ} \mathrm{C}$, and under low As pressure of $0.1 \mathrm{~atm}$. The growth rate under these conditions was about $2 \mathrm{~mm} /$ day. Small amount of $\mathrm{Yb}(0.01 \mathrm{~mol} \%)$ was added to gallium solution as a getter in order to reduce the crystal contamination with oxygen and other group VI elements. After 30 days of crystallization the process was interrupted owing to a break-down of power supply and the crystal was cooled down at a high rate of about $6 \mathrm{~K} / \mathrm{min}$. The resulting crystal of $30 \mathrm{~mm}$ diameter and $70 \mathrm{~mm}$ length was composed of monocrystalline grains of about $10 \mathrm{~mm}$ in diameter.

Several wafers were cut perpendicular to the growth direction (predominantly the $\langle 111\rangle$ crystallographic axis) and used for characterization by means of Hall effect and deep level transient spectroscopy (DLTS). DLTS measurements were performed employing both $\mathrm{Au}$-evaporated Schottky diodes and $\mathrm{Zn}$-diffused $p^{+} n$ junctions.

The crystal was $n$-type with the electron concentration of $3 \times 10^{15} \mathrm{~cm}^{-3}$ as determined by Hall effect and capacitance versus bias voltage measurements on the Schottky diodes. Selective etching of the crystal with AB etch [2] revealed large dislocation density of about $5 \times 10^{6} \mathrm{~cm}^{-2}$, likely as a result of the high post-growth cooling rate.

\section{DLTS results and discussion}

DLTS measurements revealed no impurity deep levels in concentration exceeding the detection limit of $10^{11} \mathrm{~cm}^{-3}$. Two hole traps and one electron trap detected with DLTS (Fig. 1) were attributed to native defects.

A couple of hole traps, referred to as $A$ and $B$, with activation energies, respectively, $0.40 \mathrm{eV}$ and $0.70 \mathrm{eV}$ above the valence band edge are commonly observed in LPE-grown GaAs [3, 4]. The traps were assigned to native point defects related to excess gallium in the crystal. Detailed investigation of the traps, appearing mostly in equal concentrations, led Wang et al. [4] to the conclusion that they are associated with different charge states of the same defect, likely the $\mathrm{Ga}_{\mathrm{As}}$ antisite.

These two traps were expected to occur also in our SSD crystal grown under Ga-rich conditions. In fact, the trap $B$ was the dominant deep level present in concentration of $3 \times 10^{14} \mathrm{~cm}^{-3}$ in the investigated crystal. However, the trap $A$ was not revealed in the DLTS spectrum (Fig. 1a) what calls in question a validity of the assignment of the two levels to the same defect.

Another hole trap observed at $E_{\mathrm{v}}+0.37 \mathrm{eV}$ and an electron trap at $E_{\mathrm{c}}-$ $0.68 \mathrm{eV}$, labelled HD1 and ED1 in Fig. 1, were attributed to electron states of dislocations. These two traps were recently revealed in plastically deformed $p$ - and $n$-type crystals, respectively, and identified as core states of $\alpha$ (HD1) and $\beta$ (ED1) dislocations $[5,6]$. In the present investigation an existence of the both traps were, for the first time, evidenced in the same crystal. The particular property of these traps is that the amplitudes of their DLTS peaks do not saturate but rise linearly with the logarithm of filling time (Fig. 2). Such dependence, which is characteristic of dislocations, was interpreted in the model of barrier-limited capture rate [5]. 


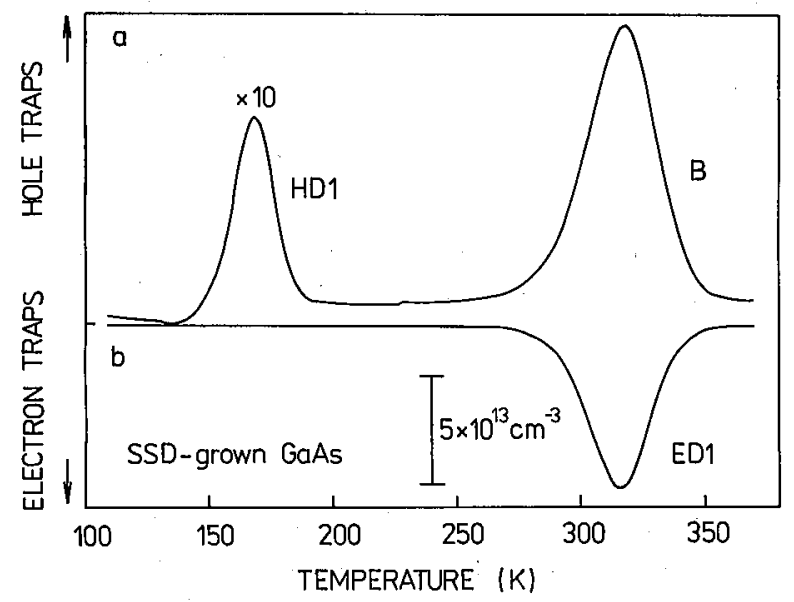

Fig. 1. DLTS spectra of the SSD-grown GaAs crystal recorded at the rate window $6.3 \mathrm{~s}^{-1}$ and the filling-pulse duration $200 \mu \mathrm{s}$. (a) Hole emission spectrum obtained with $p^{+} n$ junction. (b) Electron emission spectrum obtained with Schottky diode. Note the scale expansion for the HD1 peak.

In conclusion, it is worth noting that low concentration of $A$ traps in SSD-grown GaAs can provide for the high luminescence efficiency of this material since

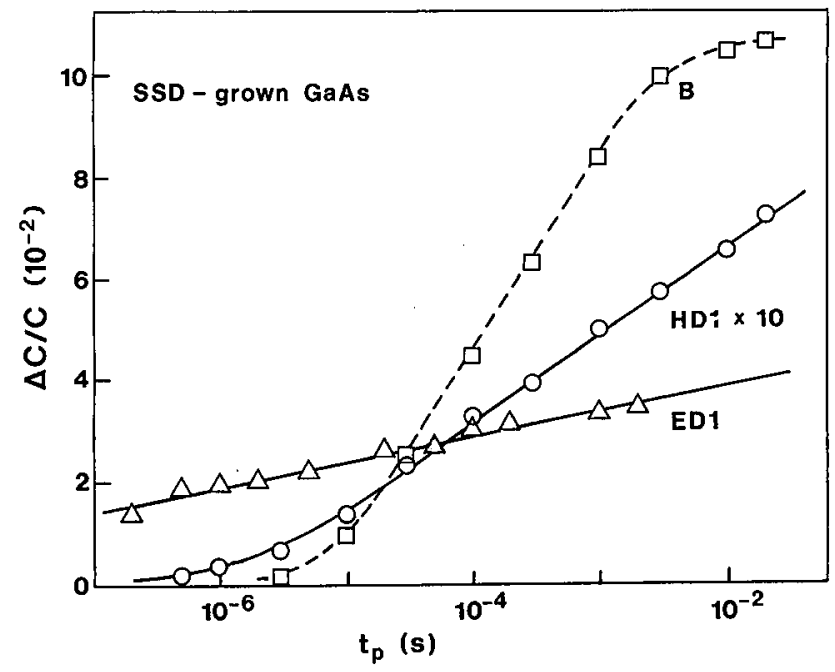

Fig. 2. DLTS peak amplitudes as a function of the filling-pulse duration. The solid lines are fitted to the data points for HD1 and ED1 according to the model of barrier-limited capture rate [5]. Note the scale expansion for the HD1 trap. 
actually the $A$ traps are responsible for degrading the minority carrier lifetime associated with nonradiative recombination in high purity LPE-grown GaAs [7].

\section{Acknowledgements}

The authors are very grateful to A. Piotrowska and E. Kaminiska (Institute of Electron Technology, Warszawa) for the preparation of the $p-n$ junctions and ohmic contacts to GaAs samples.

\section{References}

[1] T. Kobayashi, J. Osaka, J. Cryst. Growth 67, 319 (1984).

[2] M.S. Abrahams, C.J. Buiocchi, J. Appl. Phys. 36, 2855 (1965).

[3] D.V. Lang, R.A. Logan, J. Electron. Mater. 4, 1053 (1975).

[4] Z.-G. Wang, L.-A. Ledebo, H.G. Grimmeiss, J. Phys. C 17, 259 (1984).

[5] T. Wosiński, J. Appl. Phys. 65, 1566 (1989).

[6] T. Wosiński, in Defect Control in Semiconductors, Ed. K. Sumino, North-Holland, Amsterdam 1990, p. 1465.

[7] A.R. Peaker, B. Hamilton, Chemtronics 3, 194 (1988). 